\title{
A Note on Primitivity of Ideals in Skew Polynomial Rings of Automorphism Type
}

\author{
Edilson Soares Miranda \\ Departamento de Ciências, Centro de Ciências Exatas, Universidade Estadual de Maringá, 87360-000 Goioerê, PR, Brazil \\ Correspondence should be addressed to Edilson Soares Miranda; esmiranda@uem.br
}

Received 4 March 2016; Accepted 11 May 2016

Academic Editor: Kaiming Zhao

Copyright ( 2016 Edilson Soares Miranda. This is an open access article distributed under the Creative Commons Attribution License, which permits unrestricted use, distribution, and reproduction in any medium, provided the original work is properly cited.

We extend results about primitive ideals in polynomial rings over nil rings originally proved by Smoktunowicz (2005) for $\sigma$ primitive ideals in skew polynomial rings of automorphism type.

\section{Introduction}

Throughout this paper $R$ denotes an associative ring but does not necessarily have an identity element and $\sigma: R \rightarrow R$ an automorphism of $R$, unless otherwise stated. We denote by $R[x ; \sigma]$ the skew polynomial rings of automorphism type whose elements are polynomials $\sum_{i=0}^{n} a_{i} x^{i}, a_{i} \in R$, for every $i \geq 0$, with usual addition and the following multiplication: $x a=\sigma(a) x$ for all $a \in R$.

A ring $R$ is said to be a Jacobson ring if every prime ideal of $R$ is an intersection of (either left or right) primitive ideals of $R$. In [1], Smoktunowicz proved that if $R$ is a nil ring and $I$ an ideal of $R[x]$, then $R[x] / I$ is Jacobson radical if and only if $R[x] / I^{\prime}[x]$ is Jacobson radical, where $I^{\prime}$ is the ideal of $R$ generated by coefficients of polynomial from $I$. Also if $R$ is a nil ring and $I$ is a primitive ideal of $R[x]$, then $I=M[x]$ for some ideal $M$ of $R$ and affirmative answer to this question is equivalent to the Köthe conjecture. Our main results state that if $R$ is a nil ring and $I$ an ideal of $R[x ; \sigma]$, then $R[x ; \sigma] / I$ is $\sigma$-Jacobson radical if and only if $R[x ; \sigma] / I^{\prime}[x ; \sigma]$ is $\sigma$-Jacobson radical, where $I^{\prime}$ is the ideal of $R$ generated by coefficients of polynomial from $I$. Also if $R$ is a nil ring and $I$ is a $\sigma$-primitive ideal of $R[x ; \sigma]$, then $I=M[x ; \sigma]$ for some ideal $M$ of $R$. This result includes, as particular cases, all the above results.

Now we recall some terminology and results; see [2-4]. A right ideal $Q$ of a ring $R$ is called modular in $R$ if and only if there exists an element $b \in R$ such that $a-b a \in Q$ for every $a \in R$. An ideal $I$ of a ring $R$ is said to be a $\sigma$-invariant if and only if $\sigma(I)=I$. An ideal $P$ of $R$ is said to be a right $\sigma$ primitive in $R$ if and only if there exists a modular maximal right ideal $\sigma$-invariant $Q$ of $R$ such that $P$ is the maximal ideal contained in $Q$. For $f \in R[x ; \sigma], \operatorname{deg}(f)$ denotes the degree of $f$ and $\operatorname{lc}(f)$ the leading coefficient of $f$.

\section{Results}

We begin with the following results that extend ([1, Lemma 1]) and the proof is also similar to the one in the paper.

Lemma 1. Let $R$ be a ring, $J$ a right ideal of $R, f \in J[x ; \sigma], Q$ a right ideal of $R[x ; \sigma]$, and $b \in R[x ; \sigma]$ such that $a-b a \in Q$ for every $a \in R[x ; \sigma]$. If $b-f x \in Q$, then, for every $i \geq 1$, there are $f_{i} \in J[x ; \sigma]$ such that $b-f_{i} x^{i} \in Q$ and $\operatorname{deg}\left(f_{i}\right) \leq \operatorname{deg}(f)$.

Proof. We proceed by induction on $n$. If $n=1$, we put $f_{1}=f$. Suppose the lemma holds for some $n \geq 1$. Let

$$
f_{n}=a_{0}+a_{1} x+\cdots+a_{k} x^{k} \in J[x ; \sigma],
$$

with $b-f_{n} x^{n} \in Q$ and $k \leq \operatorname{deg}(f)$. Consider

$$
f_{n+1}=f \sigma\left(a_{0}\right)+a_{1}+a_{2} x+\cdots+a_{k} x^{k-1} \in J[x ; \sigma] .
$$

Since $b-f x \in Q$, then $f x=b+q, q \in Q$. Thus

$$
b-f_{n+1} x^{n+1}=b-f_{n} x^{n}+\left(a_{0}-b a_{0}\right) x^{n}-q a_{0} x^{n} \in Q .
$$


We denote by $R^{1}$ the usual extension of $R$ to a ring with identity and by $\sigma$ again the natural extension of $\sigma$ to $R^{1}$.

The next lemma extends ([1, Lemma 2]).

Lemma 2. Let $I$ be an ideal of $R[x ; \sigma]$ with $\sigma(I)=I$ and $J a$ right ideal of $R$ with $\sigma(J)=J$. Consider $p=a_{0}+a_{1} x+\cdots+$ $a_{k} x^{k} \in I, k>0$, and

$$
U=\sum_{i \in \mathbf{Z}} J[x ; \sigma] \sigma^{i}\left(a_{k}\right) R^{1}[x ; \sigma] .
$$

(i) If $h \in U^{l}, l \geq 1$, and $\operatorname{deg}(h) \geq k$, then there exists $g \in U^{l-1}$ such that $h-g \in I$ and $\operatorname{deg}(g)<\operatorname{deg}(h)$.

(ii) Let $Q$ be a right ideal of $R[x ; \sigma], b \in R[x ; \sigma]$ such that $a-b a \in Q$ for every $a \in R[x ; \sigma]$, and $I \subset Q$. If $b-f x \in$ $Q$ with $f \in J[x ; \sigma], \operatorname{deg}(f) \geq 1$, and $b-g \in Q$, where $g \in U^{\operatorname{deg}(f)}$, then, for every $i>\operatorname{deg}(g)$, there exists $g_{i} \in J[x ; \sigma]$ such that $b-g_{i} x^{i} \in Q$ and $\operatorname{deg}\left(g_{i}\right)<k$.

Proof. (i) Let $h=c_{0}+c_{1} x+\cdots+c_{t} x^{t} \in U^{l}, c_{t} \neq 0$, and $k \leq t$. We can write

$$
c_{t}=\sum_{j=0}^{m^{\prime}} \alpha_{j} \beta_{j}, \quad \alpha_{j} \in U, \beta_{j} \in U^{l-1} .
$$

Then

$$
\alpha_{j}=\sum_{i=0}^{n_{j}} t_{j i} \sigma^{q_{j i}}\left(a_{k}\right) u_{j i}, \quad t_{j i} \in J, u_{j i} \in R^{1}, q_{i j} \in \mathbb{Z} .
$$

Hence

$$
c_{t}=\sum_{j=0}^{m^{\prime}} \sum_{i=0}^{n_{j}}\left(t_{j i} \sigma^{q_{j i}}\left(a_{k}\right) u_{j i} \beta_{j}\right)=\sum_{i=0}^{m} p_{i} \sigma^{l_{i}}\left(a_{k}\right) e_{i} q_{i}
$$

with $p_{i} \in J, e_{i}, q_{i} \in R^{1}, q_{i} \in U^{l-1}$, and $l_{i} \in \mathbb{Z}$. Put

$$
\begin{aligned}
g= & h-c_{t} x^{t} \\
& +\sum_{i=0}^{m} p_{i}\left(\sigma^{l_{i}}(p)-\sigma^{l_{i}}\left(a_{k}\right) x^{k}\right) \sigma^{-k}\left(e_{i} q_{i}\right) x^{t-k} .
\end{aligned}
$$

Therefore

$$
g-h=\sum_{i=0}^{m} p_{i}\left(\sigma^{l_{i}}(p)\right) \sigma^{-k}\left(e_{i}\right) \sigma^{-k}\left(q_{i}\right) x^{t-k} \in I .
$$

Since $J[x ; \sigma] U^{l-1} \subset U^{l-1}$ and $h \in U^{l-1}$, then $g \in U^{l-1}$ and $\operatorname{deg}(g)<\operatorname{deg}(h)$.

(ii) By Lemma 1 , for every $i \geq 1$, there exists $f_{i} \in J[x ; \sigma]$ such that

$$
b-f_{i} x^{i} \in Q, \quad \operatorname{deg}\left(f_{i}\right) \leq \operatorname{deg}(f)
$$

Consider

$$
g=\sum_{j=0}^{m} c_{j} x^{j}, \quad c_{j} \in U^{\operatorname{deg}(f)} .
$$

For every $n>m$ denote

$$
h_{n}=\sum_{j=0}^{m} f_{n-j} \sigma^{n-j}\left(c_{j}\right) \in J[x ; \sigma] \cap U^{\operatorname{deg}(f)} .
$$

Note that $\operatorname{deg}\left(h_{n}\right) \leq \operatorname{deg}\left(f_{n-j}\right) \leq \operatorname{deg}(f)$; thus for every $i \geq 1$

$$
f_{i} x^{i}=b+q_{i}, \quad q_{i} \in Q .
$$

Hence

$$
b-h_{n} x^{n}=b-b \sum_{j=0}^{m} c_{j} x^{j}+\sum_{j=0}^{m} q_{n-j} c_{j} x^{j} \in Q .
$$

Because $b-g \in Q$ and $g-b g \in Q$, then $b-b g \in Q$. We have that, for every $n>t$, there exists $h_{n} \in U^{\operatorname{deg}(f)} \subseteq J[x ; \sigma]$ such that $b-h_{n} x^{n} \in Q$. If $\operatorname{deg}\left(h_{n}\right)<k$, then $h_{n}$ is the $g_{n}$ required. If $\operatorname{deg}\left(h_{n}\right) \geq k$, by first part of this lemma, there exists

$$
\lambda_{n_{1}} \in U^{\operatorname{deg}(f)-1} \subseteq J[x ; \sigma]
$$

such that $h_{n}-\lambda_{n_{1}} \in I$ and $\operatorname{deg}\left(\lambda_{n_{1}}\right)<\operatorname{deg}\left(h_{n}\right)$. Thus $b-\lambda_{n_{1}} x^{n} \in$ $Q$ for all $n>m$. If $\operatorname{deg}\left(\lambda_{n_{1}}\right)<k$, then $\lambda_{n_{1}}$ is the $g_{n}$ required. If $\operatorname{deg}\left(\lambda_{n_{1}}\right) \geq k$, using similar arguments as above, we can find $s \in \mathbb{N}$ such that

$$
\lambda_{n_{s}} \in U^{\operatorname{deg}(f)-s} \subseteq J[x ; \sigma]
$$

with $b-\lambda_{n_{s}} x^{n} \in Q$ and $\operatorname{deg}\left(\lambda_{n_{s}}\right) \leq \operatorname{deg}(f)-s<k$ for every $n>m$. Hence $\lambda_{n_{s}}$ is the $g_{n}$ required.

Let $r \in R, Q$ a right ideal of $R[x ; \sigma], \sigma(Q)=Q$, and $b \in$ $R[x ; \sigma]$ such that $a-b a \in Q$ for all $a \in R[x ; \sigma]$. Following [1] we have the following. We say that $v$ is a "good number for $r$," if, for all sufficiently large $n$, there are $f_{n} \in R[x ; \sigma]$ such that $b-r f_{n} x^{n} \in Q$ with $\operatorname{deg}\left(f_{n}\right) \leq v$. Let $A \subseteq R$; we denote

$$
\widetilde{A}=\{a \in A-Q \mid \sigma(a)-a \in Q\} .
$$

Lemma 3. Let $Q$ be a right ideal of $R[x ; \sigma]$ maximal in the set of all right ideals $\sigma$-invariants with $b \in R[x ; \sigma]$ such that $a-b a \in Q$ for all $a \in R[x ; \sigma]$. Suppose $f \in R[x ; \sigma]$ with $b-f x^{j} \in Q$ for some $j \geq 1$. If there is no right ideal J of $R$ with $\sigma(J)=J, J \nsubseteq Q$, and $J \neq R$, then there exists a positive integer $v$ and $r \in \widetilde{R}$ such that if $w \in r R[x ; \sigma]$ with $b-w x^{m} \in Q, m \geq 0$, and $\operatorname{deg}(w) \leq v$, then $l c(w) \in \widetilde{r R}, l c(w)(Q \cap R) \subseteq Q$, and $v$ is a good number for all $a \in \widetilde{r R}$.

Proof. Let $v$ be minimal positive integer such that there exists $w^{\prime}=i_{0}+i_{1} x+\cdots+r x^{v} \in R[x ; \sigma]$ and $m \geq 1$ with $b-w^{\prime} x^{m} \in Q$ and $\operatorname{deg}\left(w^{\prime}\right)=v$. It is clear that $r \notin Q$. If $c=\sigma(r)-r \notin Q$, put $g=\sigma\left(w^{\prime}\right)-w^{\prime}$ and

$$
A=\sum_{i \in \mathbf{Z}} \sigma^{i}(c) R^{1}
$$

Thus $A$ is a right ideal of $R$ with $\sigma(A)=A$ and $A \nsubseteq Q$. By assumption $A=R$, then $r=\sum_{i=0}^{s} \sigma^{q_{i}}(c) l_{i}$, where $l_{i} \in R^{1}$ and $q_{i} \in \mathbb{Z}$. Put

$$
t=w^{\prime}-\sum_{i=0}^{s} \sigma^{q_{i}}(g) \sigma^{-v}\left(l_{i}\right)
$$


Comparing the leading coefficients of $w^{\prime}$ and $\sum_{i=0}^{s} \sigma^{q_{i}}(g) \sigma^{-v}\left(l_{i}\right)$, we have that

$$
b-t x^{m} \in Q, \quad \operatorname{deg}(t) \leq v-1,
$$

which contradicts the minimality of $v$. Therefore $\sigma(r)-r \in Q$; consequently $r \in \widetilde{R}$.

Suppose that $r q \notin \mathrm{Q}$ for some $q \in R \cap Q$. Put $g^{\prime}=$ $w^{\prime} \sigma^{-v}(q) \in Q$; using similar arguments as above we can have a contradiction. Hence $r(Q \cap R) \subseteq Q$.

If there exists $w \in r R[x ; \sigma]$ with $b-w x^{j} \in Q, j \geq 0$, and $\operatorname{deg}(w) \leq v$, then using similar arguments as above we can show that $\operatorname{lc}(w) \in \widetilde{r R}$ and $\operatorname{lc}(w)(Q \cap R) \subseteq Q$. Moreover, if $a \in \widetilde{r R}$, put $B=a R+Q \cap R$; we have that $B$ is a right ideal of $R$ with $\sigma(B)=B$ and $B \nsubseteq Q$.

By assumption $B=a R+Q \cap R=R$. Thus $w^{\prime}=a w^{\prime \prime}+$ $q^{\prime}$, where $w^{\prime \prime} \in R[x ; \sigma], \operatorname{deg}\left(w^{\prime \prime}\right) \leq \operatorname{deg}\left(w^{\prime}\right)$, and $q^{\prime} \in Q$. Therefore $b-a w^{\prime \prime} x^{m} \in Q$. Consequently $v$ is a good number for all $a \in \widetilde{r R}$.

Lemma 4. Let $J$ be a right ideal $R$ with $\sigma(J)=J, J \nsubseteq Q$, and $J \neq R$ such that for all sufficiently large $n$ there are $f_{n} \in J[x ; \sigma]$ such that $b-f_{n} x^{n} \in Q$ and $\operatorname{deg}\left(f_{n}\right) \leq k$, where $Q$ is a right ideal of $R[x ; \sigma]$ and $b \in R[x ; \sigma]$ such that $a-b a \in Q$ for every $a \in R[x ; \sigma]$. Then there exists a positive integer $v$ and $r \in \widetilde{R}$ such that if $w \in r R[x ; \sigma]$ with $b-w x^{m} \in Q, m \geq 0$, and $\operatorname{deg}(w) \leq v$ one has that $l c(w) \in \widetilde{r R}, l c(w)(Q \cap R) \subseteq Q$, and $v$ is a good number for all $a \in \widetilde{r R}$.

Proof. Let $v$ be minimal positive integer such that for all sufficiently large $n$ there are $f_{n} \in J[x ; \sigma]$ such that $b-f_{n} x^{n} \in Q$ and $\operatorname{deg}\left(f_{n}\right) \leq v$. Put

$$
w^{\prime}=i_{0}+i_{1} x+\cdots+i_{v-1} x^{\nu-1}+r x^{v} \in J[x ; \sigma]
$$

with $b-w^{\prime} x^{m} \in Q, m \geq 0$, and $\operatorname{deg}\left(w^{\prime}\right) \leq v$. By Lemma 1 and minimality of $v$ we have that $r \notin Q$. Using the same ideas of Lemma 3, we have that $r \in \widetilde{R}$ and $r(Q \cap R) \subseteq Q$. Since $r R \subseteq J$, we have that the first part of lemma is satisfied.

Let $a \in \widetilde{r R} \subseteq \widetilde{J}$; we denote by $B$ the right ideal of $R$ :

$$
B=\sum_{i \in \mathbb{Z}} \sigma^{i}(a) R^{1}, \quad \sigma(B)=B, B \subseteq J, B \nsubseteq Q .
$$

For sufficiently large $n$ there are $g_{n} \in B[x ; \sigma] \subseteq J[x ; \sigma]$ such that $b-g_{n} x^{n} \in Q$ and $\operatorname{deg}\left(g_{n}\right) \leq v$. Put

$$
g_{n}=c_{n_{0}}+c_{n_{1}} x+\cdots+c_{n_{v}} x^{v} \in B[x ; \sigma] .
$$

For every $0 \leq j \leq v$ we have that $c_{n_{j}}=\sum_{i=0}^{m_{j}} \sigma^{q_{n_{i}}}(a) l_{n_{i}}$, where $l_{n_{i}} \in R^{1}$ and $q_{n_{i}} \in \mathbb{Z}$. Consequently

$$
c_{n_{j}}=\sum_{i=0}^{m_{j}}\left(\sigma^{q_{n_{i}}}(a)-a\right) l_{n_{i}}+a \sum_{i=0}^{m_{j}} l_{n_{i}} .
$$

Since $a \in \widetilde{r R}$, we can write

$$
c_{n_{j}}=s_{n_{j}}+r_{n_{j}}, \quad s_{n_{j}} \in Q \cap R, r_{n_{j}} \in R .
$$

Put $h_{n}=r_{n_{0}}+r_{n_{1}} x+\cdots+r_{n_{v}} x^{v}$; thus $b-a h_{n} x^{n} \in Q$. Therefore $v$ is a good number for all $a \in \widetilde{r R}$.
Lemma 5. Let $Q$ be a right ideal of $R[x ; \sigma], b \in R[x ; \sigma]$, such that $a-b a \in Q$ for all $a \in R[x ; \sigma]$ and $v$ is good number for all $a \in \widetilde{r R}$, where $r \in \widetilde{R}$. Assume that for every $w \in r R[x ; \sigma]$ with $b-w x^{m} \in Q, m \geq 0$, and $\operatorname{deg}(w) \leq v$ one has that $l c(w) \in \widetilde{r R}$ and $l c(w)(Q \cap R) \subseteq Q$. If there are $p$ and $p^{\prime} \in \widetilde{r R}$ with

$$
(\widetilde{p R}+Q \cap r R) \cap\left(\widetilde{p^{\prime} R}+Q \cap r R\right) \subseteq Q,
$$

then $v-1$ is a good number for $r$.

Proof. Since $v$ is a good number for $p$ and $p^{\prime}$, then for every sufficiently large $n$ there are $g_{n} \in p R[x ; \sigma]$ and $g_{n}^{\prime} \in p^{\prime} R[x ; \sigma]$ such that

$$
\begin{aligned}
& b-g_{n} x^{n} \in Q, \\
& b-g_{n}^{\prime} x^{n} \in Q
\end{aligned}
$$

with $\operatorname{deg}\left(g_{n}\right), \operatorname{deg}\left(g_{n}^{\prime}\right) \leq v$. Consider

$$
\begin{aligned}
& g_{n}=p_{n_{0}}+p_{n_{1}} x+\cdots+p_{n_{v}} x^{v}, \quad p_{n_{v}} \in \widetilde{p R}, \\
& g_{n}^{\prime}=p_{n_{0}}^{\prime}+p_{n_{1}}^{\prime} x+\cdots+p_{n_{v}}^{\prime} x^{v}, \quad p_{n_{v}}^{\prime} \in \widetilde{p^{\prime} R} .
\end{aligned}
$$

Since $p_{n_{v}}-p_{n_{v}}^{\prime} \in Q$, then

$$
p_{n_{v}} \in(\widetilde{p R}+Q \cap r R) \cap\left(\widetilde{p^{\prime} R}+Q \cap r R\right) \subseteq Q
$$

a contradiction.

Thus there exists sufficiently large $i \in \mathbb{N}$ such that $c=$ $p_{i_{v}}-p_{i_{v}}^{\prime} \in \widetilde{r R}$; hence $v$ is a good number for $c$. Then for all sufficiently large $n$ there are $h_{n} \in R[x ; \sigma]$ such that $b-c h_{n} x^{n} \in$ $Q$ and $\operatorname{deg}\left(h_{n}\right) \leq v$. We denote

$$
h_{n}=r_{n_{0}}+r_{n_{1}} x+\cdots+r_{n_{v}} x^{v}
$$

Consider

$$
k_{n}=c h_{n}+\left(g_{i}^{\prime}-g_{i}\right) \sigma^{-v}\left(r_{n_{v}}\right) \in r R[x ; \sigma] .
$$

Since $g_{i}^{\prime}-g_{i} \in Q$, then $b-k_{n} x^{n} \in Q$. Moreover

$$
k_{n}=c h_{n}-c r_{n_{v}} x^{v}+\sum_{j=0}^{v-1}\left(p_{i_{j}}^{\prime}-p_{i_{j}}\right) x^{j} \sigma^{-v}\left(r_{n_{v}}\right) \text {. }
$$

Consequently $v-1$ is a good number for $r$.

The following theorem extends ([1, Theorem 1]).

Theorem 6. Let $R$ be a nil ring and let $I$ be a $\sigma$-primitive ideal in $R[x ; \sigma]$. Then $I=I^{\prime}[x ; \sigma]$, where $I^{\prime}$ is an ideal $\sigma$-invariant of $R$.

Proof. Assume by contradiction that there are $a_{0}, a_{1}, \ldots, a_{k} \in$ $R$ with

$$
a_{0}+a_{1} x+\cdots+a_{k} x^{k} \in I, \quad a_{k} \notin I
$$


Since $I$ is a $\sigma$-primitive ideal in $R[x ; \sigma]$, there is a right ideal $Q$ of $R[x ; \sigma]$ with $\sigma(Q)=Q$ and $b \in R[x ; \sigma]$ such that $a-b a \in Q$ for all $a \in R[x ; \sigma]$. Moreover $Q$ is a maximal in the set of right ideals $\sigma$-invariants and $I$ is the maximal ideal contained in $Q$. We have that $R[x ; \sigma] x \nsupseteq Q$; otherwise $b \in R$, which is impossible because $R$ is a nil ring. By definition of $Q$ it follows that $R[x ; \sigma] x+Q=R[x ; \sigma]$.

If $b-h x^{i} \in Q$ for some $i \geq 0$ with $h \in R[x ; \sigma]$, then $\operatorname{deg}(h) \geq 1$. In fact, if $h \in R$, let $t \geq 1$ be the minimal positive integer with respect to $h^{t} \in Q$. Thus $\left(b-h x^{i}\right) \sigma^{-i}\left(h^{t-1}\right) \in Q$. Then $b \sigma^{-i}\left(h^{t-1}\right) \in Q$; hence $\sigma^{-i}\left(h^{t-1}\right) \in Q$. Consequently $h^{t-1} \in Q$, a contradiction.

Let $J$ be a right ideal of $R$ with $\sigma(J)=J$ and $J \nsubseteq Q$. We have that $J[x ; \sigma] x+Q=R[x ; \sigma]$. There exists $f \in J[x ; \sigma]$ such that $b-f x \in Q$. Consider

$$
U=\sum_{i \in \mathbb{Z}} J[x ; \sigma] \sigma^{i}\left(a_{k}\right) R^{1}[x ; \sigma] .
$$

Since $I$ is an ideal $\sigma$-prime and $a_{k} \notin I$, then $U \nsubseteq I$. Consequently $U \nsubseteq Q$, because $I$ is the maximal ideal contained in $Q$. Then $U^{\operatorname{deg}(f)}+Q=R[x ; \sigma]$. There exists $g^{\prime} \in U^{\operatorname{deg}(f)}$ such that $b-g^{\prime} \in Q$. By Lemma 2, for every $i \geq \operatorname{deg}\left(g^{\prime}\right)$, there are $g_{i}^{\prime} \in J[x ; \sigma]$ such that $b-g_{i}^{\prime} x^{i} \in Q$ and $\operatorname{deg}\left(g_{i}^{\prime}\right)<k$. Lemmas 3 and 4 imply that there are $r^{\prime} \in \widetilde{R}$ and $v^{\prime} \geq 1$ such that if $w \in r^{\prime} R[x ; \sigma]$ with $b-w x^{m} \in Q, m \geq 1$, and $\operatorname{deg}(w) \leq v^{\prime}$, then $\operatorname{lc}(w) \in \widetilde{r^{\prime} R}$ and $\operatorname{lc} w(Q \cap R) \subseteq Q$. Moreover $v^{\prime}$ is a good number for all $a \in \widetilde{r^{\prime} R}$. Let $v$ be minimal such that $v$ is a good number for all $a \in \widetilde{r^{\prime} R}$. We have that $v \leq v^{\prime}$. Let $r \in \widetilde{r^{\prime} R}$. Since $v$ is a good number for $r$, then for sufficiently large $n$ there are $h_{n} \in R[x ; \sigma]$, such that

$$
b-r h_{n} x^{n} \in Q, \quad \operatorname{deg}\left(h_{n}\right) \leq v .
$$

Consider $f_{n}=r h_{n}$, then $b-f_{n} x^{n} \in Q$ and $\operatorname{deg}\left(f_{n}\right) \leq v$. For some $i \in \mathbb{N}$, there are $f_{i}, f_{i+1}, \ldots, f_{i+k} \in r R[x ; \sigma]$, such that $b-f_{j} x^{j} \in Q, \operatorname{deg}\left(f_{j}\right) \leq v$, and $i \leq j \leq i+k$. Put

$$
f_{j}=r a_{j_{0}}+r a_{j_{1}} x+\cdots+r a_{j_{v}} x^{v}=g_{j}+c_{j} x^{v},
$$

where $g_{j}=r a_{j_{0}}+r a_{j_{1}} x+\cdots+r a_{j_{v-1}} x^{\nu-1} \in r R[x ; \sigma]$ and $c_{j}=r a_{j_{v}}$. Since $\operatorname{deg}\left(f_{j}\right) \leq v \leq v^{\prime}$, then $c_{j} \notin Q$. Moreover,

$$
\sigma\left(c_{j}\right)-c_{j} \in Q, \quad c_{j}(Q \cap R) \subseteq Q .
$$

Since $R$ is a nil ring, consider $e_{j}=c_{j}^{n_{j}}$, where $n_{j}$ is a minimal with respect to the condition $c_{j}^{n_{j}} \notin Q$. Thus $\sigma\left(e_{j}\right)-e_{j} \in Q$ for all $i \geq 0$. We have that

$$
\begin{aligned}
f_{j} \sigma^{j}\left(e_{j}\right)= & g_{j} \sigma^{j}\left(e_{j}\right)+c_{j} \sigma^{j+v}\left(e_{j}\right) x^{v} \\
= & g_{j} \sigma^{j}\left(e_{j}\right)+c_{j}\left(\sigma^{j+v}\left(e_{j}\right)-e_{j}\right) x^{v} \\
& +c_{j} e_{j} x^{v} .
\end{aligned}
$$

Put $t_{j}=g_{j} \sigma^{j}\left(e_{j}\right) \in r R[x ; \sigma]$. Thus,

$$
f_{j} \sigma^{j}\left(e_{j}\right)-t_{j} \in Q \quad \operatorname{deg}\left(t_{j}\right) \leq v-1
$$

for every $i \leq j \leq i+k$. Since $e_{j} \in \widetilde{r R} \subseteq \widetilde{r^{\prime} R}$, if $v-1$ is not a good number for $r$, then Lemma 5 implies that

$$
\bigcap_{j=1}^{i+k}\left(\widetilde{e_{j} R}+Q \cap r R\right) \nsubseteq Q \text {. }
$$

In this case, there exists $s \in \bigcap_{j=1}^{i+k}\left(\widetilde{e_{j} R}+Q \cap r R\right)$ such that $s \notin Q$. Consequently $s-e_{j} d_{j} \in Q \cap r R, d_{j} \in R$, and $e_{j} d_{j} \in \widetilde{e_{j} R}$. Then $s \in \widetilde{r R} \subseteq \widetilde{r^{\prime} R}$. Therefore $v$ is a good number for $s$. Then for sufficiently large $n$ there are $\bar{f}_{n} \in s R[x ; \sigma]$, such that

$$
b-\bar{f}_{n} x^{n} \in Q, \quad \operatorname{deg}\left(\bar{f}_{n}\right) \leq v .
$$

Let

$$
\bar{f}_{n}=\sum_{j=0}^{v} s b_{j_{n}} x^{j}
$$

Since $b-\bar{f}_{j} x^{j} \in Q, s-e_{j} d_{j} \in Q$, and $e_{j} d_{j}-b e_{j} d_{j} \in Q$, then $\left(b-f_{j} x^{j}\right) e_{j} d_{j} \in Q$. Thus $b e_{j} d_{j}-f_{j} x^{j} e_{j} d_{j} \in Q$; hence $s-f_{j} x^{j} e_{j} d_{j} \in Q$ for every $i \leq j \leq i+k$.

$$
\bar{g}_{n}=\sum_{j=0}^{v} f_{i+v-j} \sigma^{i+v-j}\left(e_{i+v-j} d_{i+v-j} b_{j_{n}}\right) x^{i+v} \in r R[x ; \sigma] .
$$

We have that $\bar{f}_{n}-\bar{g}_{n} \in Q$. Thus $b-\bar{g}_{n} x^{n}=\left(b-\bar{f}_{n} x^{n}\right)+\left(\bar{f}_{n}-\right.$ $\left.\bar{g}_{n}\right) x^{n} \in Q$. Put

$$
\bar{h}_{n}=\sum_{j=0}^{v} t_{i+v-j} \sigma^{i+v-j}\left(d_{i+v-j} b_{j_{n}}\right) \in r R[x ; \sigma] .
$$

We can write $b-\bar{h}_{n} x^{i+v+n}$ as

$$
\begin{aligned}
b- & \sum_{j=0}^{v}\left(t_{i+v-j}-f_{i+v-j} \sigma^{i+v-j}\left(e_{i+v-j}\right)\right) \sigma^{i+v-j}\left(d_{i+v-j} b_{j_{n}}\right) \\
& \cdot x^{i+v+n}-\bar{g}_{n} x^{n} .
\end{aligned}
$$

Thus for all sufficient large $n$

$$
b-\bar{h}_{n} x^{i+v+n} \in Q, \quad \operatorname{deg}\left(\bar{h}_{n}\right) \leq v-1 .
$$

Then $v-1$ is a good number for all $r \in \widetilde{r^{\prime} R}$. This contradicts the minimality of $v$.

Recall that the $\sigma$-Jacobson radical $J_{\sigma}(R)$ of a $\operatorname{ring} R$ is defined as the intersection of all $\sigma$-primitive ideals of $R$. A ring $R$ is a $\sigma$-Jacobson radical if $J_{\sigma}(R)=R$.

Theorem 7 . Let $R$ be a nil ring and let $I$ be an ideal of $R[x ; \sigma]$. Consider $\bar{I}$ the ideal of $R$ generated by coefficients of polynomial from $I$. Then $R[x ; \sigma] / \bar{I}[x ; \sigma]$ is $\sigma$-Jacobson radical if and only if $R[x ; \sigma] / I$ is $\sigma$-Jacobson radical. 
Proof. Assume by contradiction that $R[x ; \sigma] / I$ is not $\sigma$ Jacobson radical. Then there is a $\sigma$-primitive ideal $P$ of $R[x ; \sigma] / I$ such that $P \neq R[x ; \sigma] / I$. We have that there is an ideal $K$ of $R[x ; \sigma]$ such that $P=K / I$. Therefore $K$ is a $\sigma$ primitive ideal of $R[x ; \sigma]$. By Theorem 6 , there is an ideal $\bar{P}$ of $R$ such that $K=\bar{P}[x ; \sigma]$. It is clear that $\bar{I} \subseteq \bar{P}$. Since

$$
\frac{(R[x ; \sigma] / \bar{I}[x ; \sigma])}{(\bar{P}[x ; \sigma] / \bar{I}[x ; \sigma])} \simeq \frac{R[x ; \sigma]}{K},
$$

then $\bar{P}[x ; \sigma] / \bar{I}[x ; \sigma]$ is a $\sigma$-primitive ideal, a contradiction. Using the fact that $I \subseteq \bar{I}[x ; \sigma]$, the converse follows.

Corollary 8. If $R$ is a nil ring, then the polynomial ring of type automorphism $R[x ; \sigma]$ can not be homomorphically mapped onto a $\sigma$-simple $\sigma$-primitive ring.

\section{Competing Interests}

The author declares that they have no competing interests.

\section{References}

[1] A. Smoktunowicz, "On primitive ideals in polynomial rings over nil rings," Algebras and Representation Theory, vol. 8, no. 1, pp. 69-73, 2005.

[2] E. Cisneros, M. Ferrero, and M. I. Conzles, "Prime ideals of skew polynomial rings and skew laurent polynomial rings," Mathematical Journal of Okayama University, vol. 32, pp. 61-72, 1990.

[3] N. Divinsky, Rings and Radicals, Allen and Unwin, London, UK, 1965.

[4] T. Y. Lam, A First Course in Noncommutative Rings, Graduate Texts in Mathematics, Springer, New York, NY, USA, 1991. 


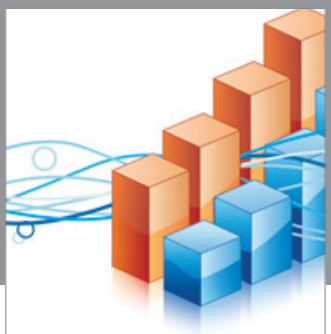

Advances in

Operations Research

vatem alat4

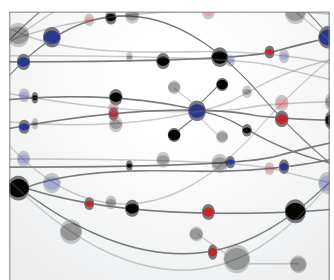

\section{The Scientific} World Journal
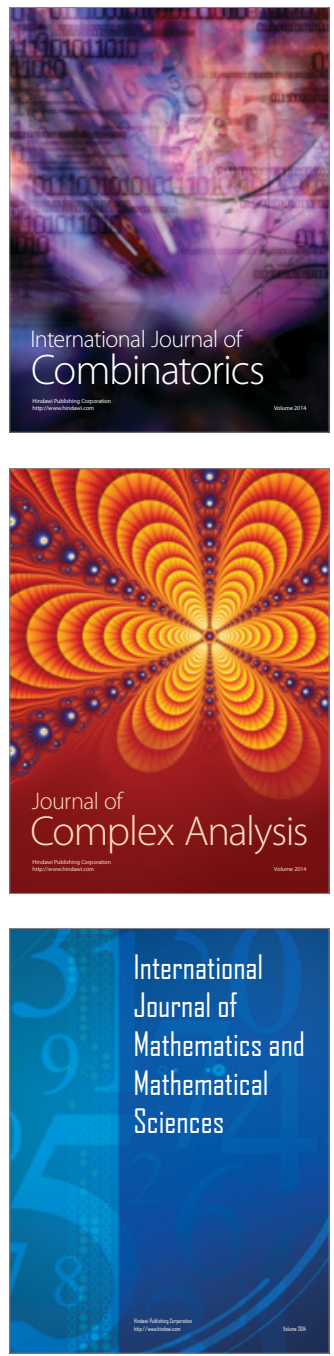
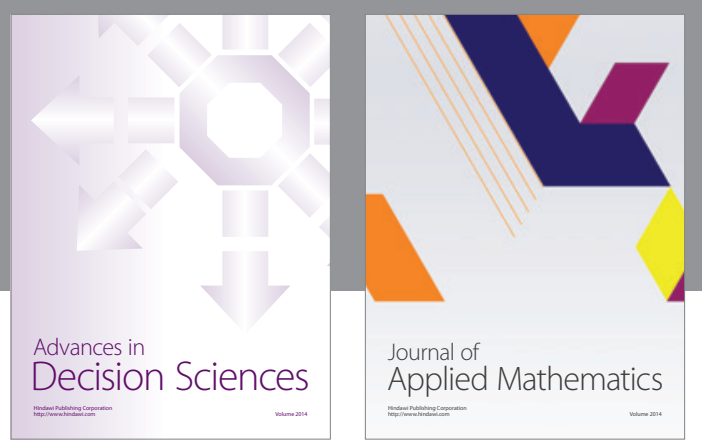

Algebra

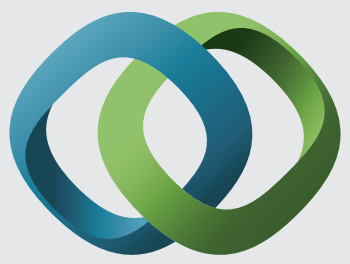

\section{Hindawi}

Submit your manuscripts at

http://www.hindawi.com
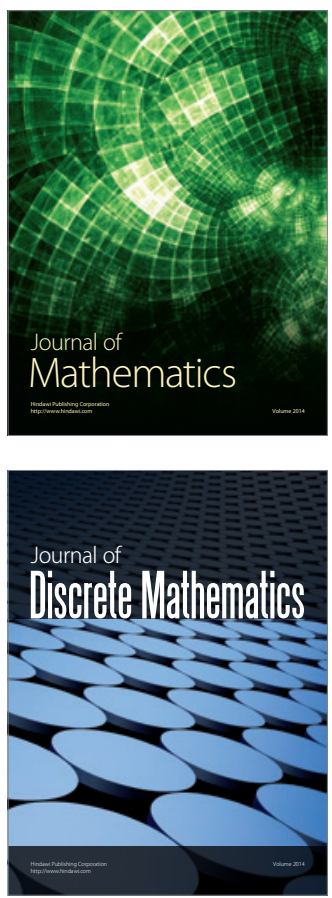

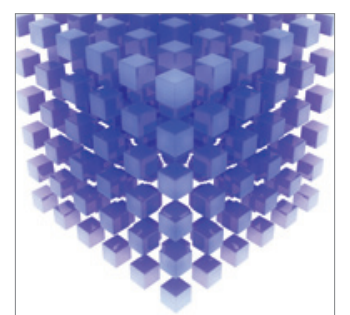

Mathematical Problems in Engineering
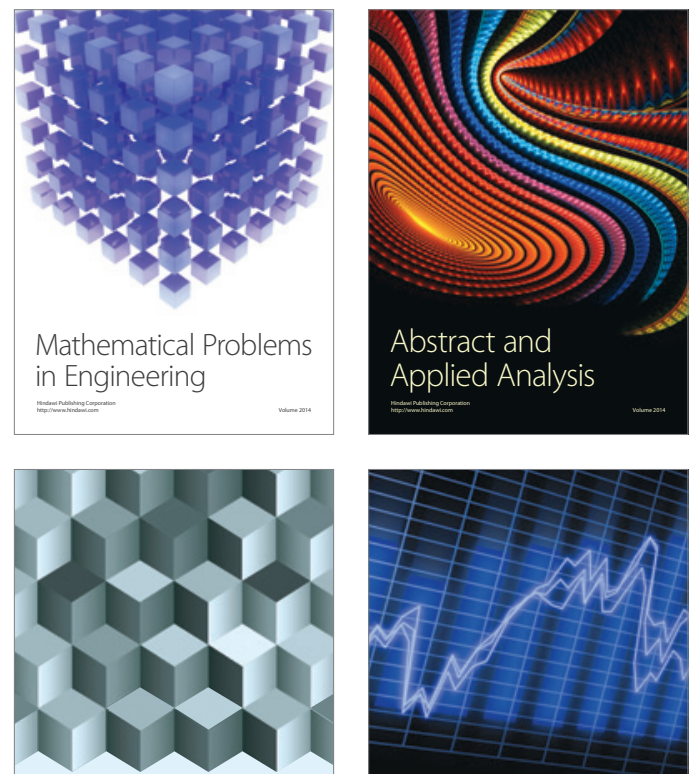

Journal of

Function Spaces

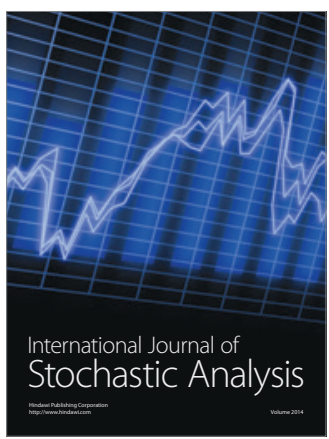

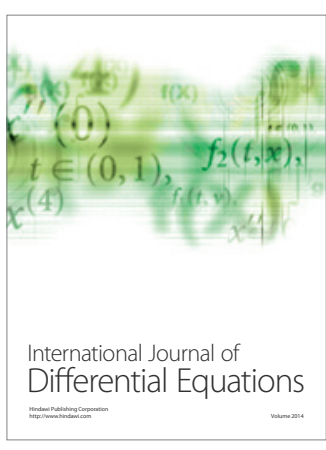
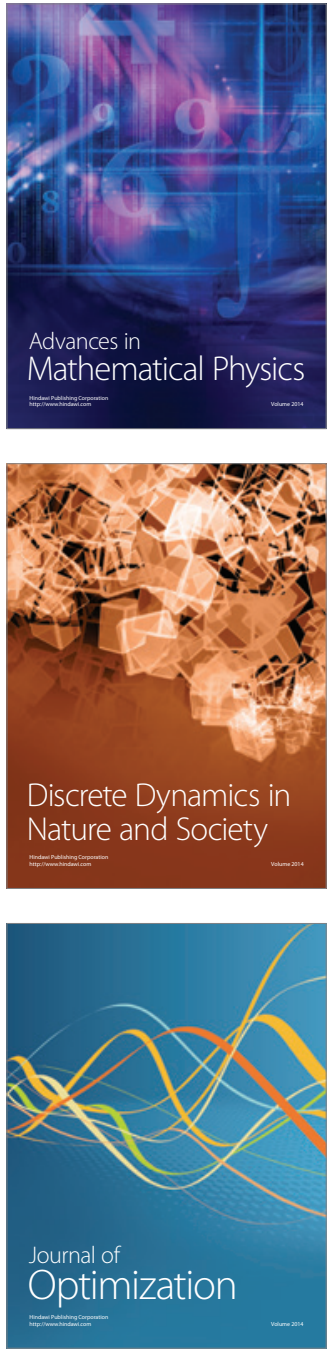